\title{
The Simulation Model of a Sliding Contact
}

\author{
Alexander Ilyin, Igor Plokhov, Andrey Isakov \\ Pskov State University, Faculty of Electrical Engineering, Department of Drive and \\ Automation Systems. Address: Lenina 8, Pskov, 180000, Russia.
}

\begin{abstract}
This paper deals with the brush slip ring contact modeling. Approaches the sliding contact problem with the synergy theory. The contact layer is considered using mechanical, electrical and thermal properties.

The simulation model includes algorithms of:

- generation of microscale contact surface,

- approachment of two surfaces,

- fritting and breakdown effects of oxide layer,

- extracting clusters of conducting area,

- calculating constriction resistance using fractal dimensionality,

- generation of heat caused by electrical current and mechanical friction,

- calculation of integral characteristics.
\end{abstract}

The program implementing the model may be used to optimize the contact materials before time-consuming and expensive tests.

Finally discusses the implemented model and its results, problems of verification and validation.

Keywords - brush, slip ring, sliding contact, modeling.

\section{INTRODUCTION}

Improvement of reliability of electrical machines is related to progress of fundamental and applied study in diagnostics and prediction of its technical condition. Methods of mathematical computational simulation of dynamic processes are developing nowadays.

Sliding contacts are widely used in engineering despite the trend towards non-contact methods of transmission of electrical current. In some cases, the use of brush-contact devices is appropriate due to their low cost and continuity of operations.

Up to now there is no consensus on the adequate modeling of the main properties and characteristics of sliding electrical contact, due to a wide variety and complexity of electric friction interaction processes.

Therefore, theoretical and experimental research in this area increasing range of scientific ideas about electrical contacts which provides new ways of modeling is topical problem.

Particular attention should be given to computational simulation modeling, intend to obtain the integral characteristics of the site to the parameters of current collection sliding contact pairs and external influences.

This approach can significantly reduce the amount of expensive practical experiments in the study of the characteristics of different contact pairs.

Dynamic processes occurring in sliding electrical contact can be divided into the following categories:
1. mechanical,
2. electromagnetic,
3. thermal,
4. chemical

These processes are bonded directly or through intermediate functional blocks.

The processes occurring directly in the contact layer include:

- frictional interaction processes of microscale contact surfaces;

- the evolution of the contact conductance in the contact layer;

- the evolution of nonsteady temperature field in the contact layer;

- oxidation-reduction and electrolytic reactions.

In addition there are external effects:

- brush fluctuations caused by kinematic and dynamic disturbances;

- oscillating processes of dynamic electrical current distribution among the parallel contacts;

- overall heating of contact pairs.

The objective of the developing model is calculation of dynamic electrical current transmission processes in the intermediate layer of sliding contact [1].

\section{SYSTEM APPROACH TO THE SLIDING CONTACT MODELLING}

When two bodies are pressed together, the contact of its surfaces can be described as a system of discrete elements. The intermediate layer can be considered as a "third body" consisting of a number of interrelated areas, located in the plane equidistant from the two contacting surfaces. Thus the intermediate layer is replaced by an abstract object that allows using a purely mathematical definitions and operations. 
Divide the intermediate layer on the set of elementary parts of the same area $\Delta S$, which can be called the contact elements. They are located in grid with increments $\Delta \mathrm{x}$ by the axis $\mathrm{X}$ and with increments $\Delta y$ by the axis $Y$. In the practice of computational modeling it takes $\Delta \mathrm{x}=\Delta \mathrm{y}$.

The contact elements of the system have different properties depending on the way of current transmission through them and are united in the subsystems. The subsystems present a flock of fractal clusters of different nature. The cluster system is a projection plane of contact conductivity of various types phenomena in the intermediate layer, as conductive objects between two microscale surfaces formed by various types of connection (direct contact, contact through the oxide film, contact through wear products and contact through a low-temperature plasma). The conducting clusters specify these properties in the form of appropriate mathematical models to the contact elements.

Introducing the intermediate layer, the binary relations of two sets (microscale surfaces) are replaced by unary relations in the conductive properties of the contact layer clusters. This approach opens the possibility of system simulation of the electrofrictional interaction and study its integral characteristics within the synergetic open system.

Thus a generalized model of a sliding electrical contact appears as a regular set of finite elements in the intermediate layer combined with the properties and relationship bonds. The contact elements of the same type form the clusters. The contact element properties appear when external influences are applied to the contact pair.

This system is an evolutionary, because for the first time application of the external influences the conductive structure of clusters is minimal. The evolution of the conducting clusters is a result of changes in the type and magnitude of conduction in the associated contact elements. Changing of the cluster parameters are determined by the values of external influences, the evolutionary properties of the contact elements and their evolutionary relationships [2].

The main external factors at the input of the "sliding electrical contact" can be divided into the following categories:

1) controlled and regulated; these include steady components of electric voltage on the contact pair and applied pressure, the velocity of contact pairs relative movement;

2) controlled but nonregulated; these include variable components of the voltage and applied pressure, temperature and chemical composition of the environment, the microscale of contact surfaces;

3) uncontrolled (disturbances).
III THE SYNERGETIC MODEL OF ELECTROFRICTIONAL INTERACTION

The intermediate layer can be represented as a set of contact elements placed in the regular grid. Each contact element can be described with a state vector V.

The basic components of the state vector of the contact element is divided into the following classes.

1. Mechanical $\mathrm{V}_{\mathrm{M}}$ :

- contact stiffness $\mathrm{C}_{\mathrm{C}}$;

- contact damping $\mathrm{K}_{\mathrm{C}}$;

- contact convergence $\mathrm{Y}_{\mathrm{C}}$;

- contact force $\mathrm{F}_{\mathrm{C}}$;

- microhardness q;

- density $\rho_{\mathrm{M}}$;

- coefficient of boundary friction $\mathrm{k}_{\mathrm{FR}}$.

2. Electrical $\mathrm{V}_{\mathrm{E}}$ :

- voltage U (the same for all contact elements);

- fritting voltage $\mathrm{U}_{\mathrm{F}}$;

- electrical conductivity $\rho_{\mathrm{E}}$ or R;

- contact capacity $\mathrm{C}_{\mathrm{E}}$;

- the current I through the contact element.

3. Thermal $\mathrm{V}_{\mathrm{T}}$ :

- heat capacity $\mathrm{C}_{\theta}$;

- thermal conductivity k;

- melting temperature $\theta_{\mathrm{M}}$;

- evaporation temperature $\theta_{\mathrm{Ev}}$;

- instant temperature $\theta$;

4. Chemical $\mathrm{V}_{\mathrm{H}}$ :

- vector of chemical composition $\hbar$;

- intensity of the basic chemical reactions $V_{\hbar}$.

The contact stiffness of the element is nonlinear function of temperature. The exact definition of this dependence is associated with considerable difficulties. Therefore, it can be defined by the following considerations.

The contact stiffness has a weak temperature dependence in the range from room temperature to the temperature of recrystallization $\left(\theta_{\mathrm{R}}\right)$. In the range from $\theta_{R}$ to the melting temperature $\theta_{M}$ softening of the material starts and the gradual loss of hardness occurs (up to $C_{M}=0$ ). After that, there is only a viscous drag force proportional to the velocity of convergence $\mathrm{Y}_{\mathrm{C}}$ changing and determined by damping factor $\mathrm{K}_{\mathrm{C}}$. Changing the stiffness to zero is in the range $] \theta_{\mathrm{R}}, \theta_{\mathrm{M}}[$ so:

$$
C_{C}=\left\{\begin{array}{l}
C_{C N} \forall \theta \leq \theta_{R}, \\
\left.C_{C N} \cdot \frac{\theta_{M}-\theta}{\theta_{M}-\theta_{R}} \forall \theta \in\right] \theta_{R}, \theta_{M}[, \\
0 \forall \theta \geq \theta_{M},
\end{array}\right.
$$

where $\mathrm{C}_{\mathrm{CN}}$ - the nominal stiffness of the element. [3]

Recrystallization temperature of copper is about $190^{\circ} \mathrm{C}$, of iron $500^{\circ} \mathrm{C}$. The strength characteristics of graphite increase with temperature. Decreasing of 
the mechanical strength of the contact material (softening) starts at the contact surface at about 0.3 of the melting temperature [4].

The contact force $\mathrm{F}_{\mathrm{C}}$ can be presented as two components $\mathrm{F}_{\mathrm{C}}=\mathrm{F}_{1}+\mathrm{F}_{2}$. The first is the reaction of the contact stiffness to convergence and viscous drag force

$$
F_{1}=C_{C} Y_{C}+K_{C} \frac{d Y_{C}}{d t}
$$

and the second is the total electrodynamic force

$$
F_{2}=\frac{\mu_{0} I^{2}}{4 \pi} \ln \frac{R_{0}}{r}+\frac{\mu_{0} I^{2}}{6 \pi},
$$

where $\mathrm{R}_{0}, \mathrm{r}-$ the equivalent radius of the direct current transmission area and the radius of the contact; $\mu_{0}$ - the magnetic conductivity.

The density $\rho$ is defined in terms of mass $m_{i}$ of contact materials in the fixed volume of the contact element

$$
\rho=\frac{1}{V_{C E}} \sum_{i=1}^{n} m_{i}
$$

Electrical resistance or conductivity of the contact element is described with complex model. The model is a system of submodels which work alone or in groups depending on the conditions. The submodels describe the different types of conduction through contact element: conduction through the oxide films, conduction through the gas gaps, direct conduction, conduction through the wear particles.

a) Conduction through the oxide films.

The films are formed by the interaction of a contact material with oxygen, sulfur, nitrogen and other substances and conditionally subdivided into thin and thick.

Adhesive films have a thickness of 9-30 $\AA$ and are the result of adsorption of oxygen molecules on the contact surface. Dependence of the tunneling resistance on the film thickness $d$ is practically linear

$$
\sigma_{f}=\rho_{f} d
$$

where $\rho_{\mathrm{f}}-$ linear resistivity of the film material.

Thick films (tarnishing films) divided into oxide and sulphide. Maximum thickness of the oxide film is a function of time and temperature. For example, for copper this value is given by the empirical relation

$$
\Delta h=\sqrt{100+t 10^{8,2-\frac{1310}{\theta}}} .
$$

Fritting of the thick films takes place when the electric field $E \approx 10^{6} \mathrm{~V} / \mathrm{m}$. Fritting voltage $U_{F}=E \Delta h$ determine for each contact element. In the breakdown of the film forms a conductive metal bridge, which changes the conduction submodel of the direct conduction for the contact element.

b) Conduction through the gas gaps.

For plasma conductivity is a function of temperature. Calculations show that the value of the gas component in the total contact conductance becomes significant only at temperatures above $4000 \mathrm{~K}$. Only contact elements located in the place of the intense "heat flashes" have this type of conductivity. For the contact elements that have a lower temperature it is possible to neglect the gas conductivity without loss of accuracy.

c) Direct conduction

Direct conduction occurs by direct contact of the roughness of contacted bodies surfaces. In addition the contact elements affected by fritting have similar conductivity.

The value of the electric current flowing through direct contact is determined by constriction resistance. For $n$ spots of radius $a$ the contact resistance is given by Holm equation

$$
R_{C}=\rho\left(\frac{1}{2 \cdot n \cdot a}+\frac{1}{2 \cdot a_{k}}\right),
$$

where $a_{k}$ is the equivalent radius of all spots area [5]. Greenwood improved the second part [6]

$$
R_{C}=\rho\left(\frac{1}{2 \cdot n \cdot a}+\frac{16}{3 \cdot \pi^{2} \cdot a_{k}}\right)
$$

Plokhov suggested to define the constriction resistance of the conducting cluster as

$$
R_{C L}=\frac{\rho}{2 \cdot a_{C L} \cdot D_{F}},
$$

where $\mathrm{a}_{\mathrm{CL}}$ is the equivalent radius of the cluster; $\mathrm{D}_{\mathrm{F}}$ is the fractional dimensionality of cluster. The fractional dimensionality is described in the fractal theory. The proof of the equation in general is considerably complexity. Therefore, a series of experimental studies were made to verify the above formula [1].

d) Conduction through the wear particles

With data on the size of the particles and the conductivity of its material an additional electrical resistance in the contact element can be defined. If the particle covers several contact elements then it is presented as the conglomerate of several separate particles. 
Each contact element has a heat capacity $C$ which is determined by its mass $m$ and specific heat of the material $c$

$$
C=c \cdot m, m=\Delta x^{2} \cdot \Delta h \cdot \rho,
$$

where $\Delta \mathrm{h}$ is the height of the element; $\Delta \mathrm{x}$ is the value of the grid size; $\rho$ is density of the contact element material.

The specific heat depends on the temperature of the material. This dependence of each material can be presented in the model corresponding to the approximating function. According to studies using a linear approximation is sufficient.

Redistribution of heat in the contact area due to the thermal conductivity $\mathrm{k}$, which also depends on the temperature. For most materials this dependence with sufficient accuracy can be considered as linear

$$
k=k_{\mathrm{o}} \cdot\left[1+b \cdot\left(\theta-\theta_{0}\right)\right] \text {, }
$$

where $\mathrm{k}_{0}$ is the value of thermal conductivity at $\theta_{0} ; \mathrm{b}$ is the constant determined empirically.

\section{THE SIMULATION MODEL STRUCTURE}

Basic algorithmic actions performed during simulation can be divided into the following units: a) setting the initial values of the contact elements state vector, b) generating the vector of controls and disturbances, c) modification of the contact elements state vector, d) the calculation and presentation of integral characteristics.

Together these actions can be described as a list of operations.

For block (a).

1. Generation of two-dimensional arrays of cells that define microscale of two contacting surfaces with the oxide films.

2. Setting the parameters of materials and surface films.

3. Convergence of surfaces is determined by the value of the force pushing to the contact and the contact stiffness.

4. Filling the two-dimensional array of intermediate layer, which determines the distribution of clusters of direct conduction and conduction through films.

For block (b).

1. Setting the speed of sliding and sampling time.

2. Setting step movement of microscales by reassignment of surfaces arrays.

3. Setting force and the initial convergence of microscales.

4. Specifying the electric voltage.

5. Setting the initial temperature.

For block (c).

1. Calculation of the constriction resistances of allocated clusters.
2. Calculation of conducting clusters currents.

3. Calculation of the heat generated in clusters.

4. Calculation of nonsteady temperature field.

5. Modification of the contact stiffness as a function of temperature.

6. Modification of convergence depending on the contact stiffness and contact pressure.

7. Modification of the values and types of conductivity as a function of temperature.

8. Modification of conductivity type as result of fritting.

9. Modification of the thickness of surface films by chemical, thermal, and mechanical processes.

For block (d).

1. Calculation of the total current and voltage drop on intermediate layer.

2. Determination of the average cluster temperature and of the overall contact.

3. Calculation of physical (actual) contact area.

4. Forming a dynamic voltage-current characteristics, charts of current, temperature, etc.

5. Visualization of microscales, of the temperature field and the contact conductance.

\section{CONCLUSION}

The proposed model is implemented as a computer program. The figures show examples of two-dimensional arrays visualization (Fig. 1 Fig. 3).

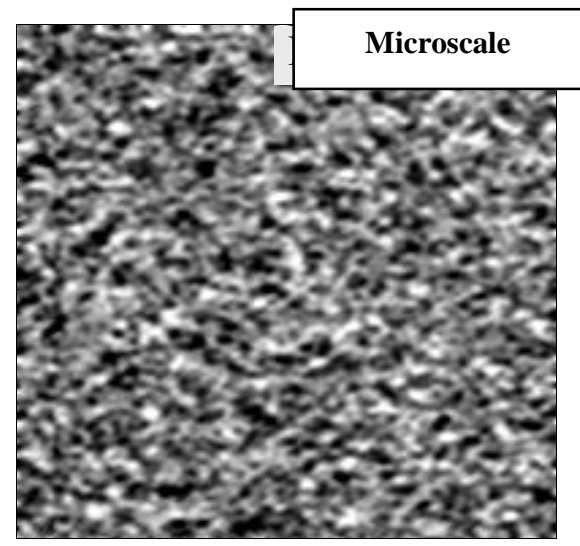

Fig. 1. Visualization of microscale contact surface

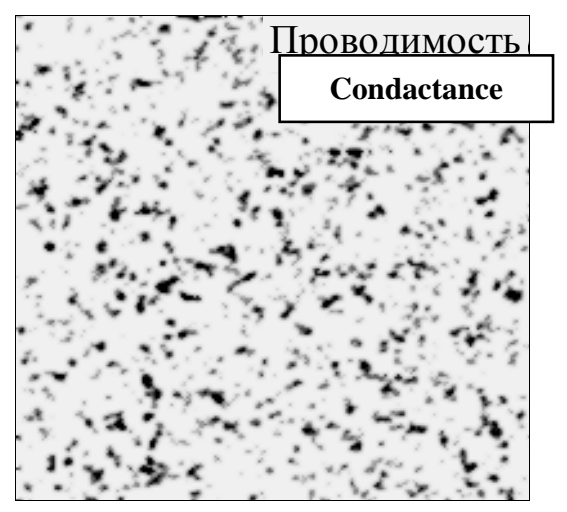

Fig. 2. Visualization of conductance in intermediate layer 


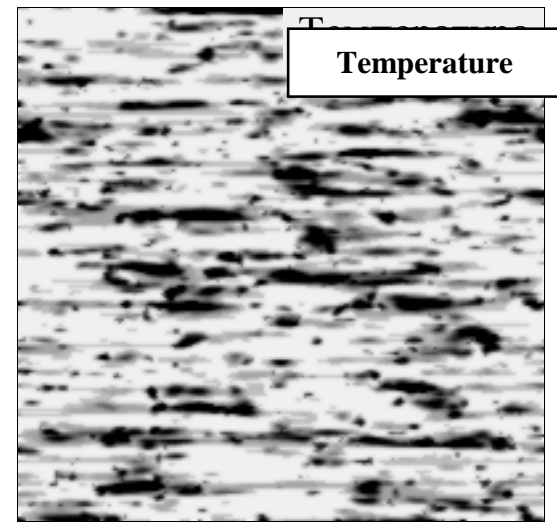

Fig. 3. Visualization of nonsteady temperature field

Currently research on the verification and validation of the simulation model is performed. An experimental stand of brush and slip ring assembly is developed. The stand allows taking the current- voltage characteristics of the brushes in various operation modes.

\section{REFERENCES}

[1] Plokhov I. V. Comprehensive diagnosis and prediction of technical state of nodes moving current collection turbogenerators. // Dissertation for doctor degree Saint Petersburg, 2001

[2] Plokhov I. V. Cluster model of electrofriction interaction (EFI) // Proceedings of the Pskov Polytechnic Institute. Saint Petersburg /Pskov: SPbSTU, 1997. \#1. P.55-57.

[3] 9. Mechanics of sliding contact / V. I. Nellin, N. Ya. Bogatyrev, L. V. Lozhkin and others - Moscow, 1966.

[4] 160. Guide to the calculation and design of high-contact parts of electrical apparatus / N. M. Adonyev, V. V. Afanasyev, V. V. Borisov and ohers - Leningrad, 1988.

[5] Holm R. Electric contacts. - Moscow, 1961.

[6] Greenwood J. A. Constriction resistance and the real area of contact. - British Journal of appl. Physics. 1966. V.17.P.1621-163. 